\title{
EDITORIAL
}

\section{Turn on, tune in, speak up}

\author{
Despite low levels of uptake and active engagement, social media and other online tools can \\ be of great benefit to microbiology researchers, and their use is to be encouraged.
}

The phrase 'turn on, tune in, drop out', popularized in the 1960s by the late American psychologist Timothy Leary, urged people to embrace cultural change and detach from existing conventions and hierarchies through the use of psychedelic drugs. Although we certainly would not encourage our readers to take any such substance (of microbial origin or otherwise), this phrase does resonate with the changing culture of science today.

The past decade has seen a revolution in the online tools available to researchers and the technology with which to access them. In addition to this, the rise of social media has changed the way that researchers can engage with these tools and, consequently, with each other. Many of these tools have become widely used in supporting individual and collaborative research efforts ${ }^{1}$. In particular, social media tools, including blogs and microblogging services such as Twitter, have dramatically changed the manner in which the results of scientific research and other issues pertinent to researchers can be communicated and discussed.

However, despite the huge potential of social media and online science, a large gap remains between our awareness of the tools and our actual use of them, even among researchers at early stages of their careers ${ }^{2,3}$. Estimates of the percentage of researchers who use any type of social media for communicating with other researchers are difficult to obtain; however, one recent survey of more than 13,500 doctoral students at higher-education institutions in the United Kingdom found that $23 \%$ follow blogs and only $9 \%$ blog themselves $^{3}$. For microblogging sites, the numbers are even lower, at $7 \%$ for following and 5\% for actively microblogging. So what explains this low level of engagement? Contributing factors probably include concerns about the authenticity and quality of information sourced from non-traditional channels ${ }^{2}$, and in some cases participation may be actively discouraged by colleagues or supervisors ${ }^{4}$. Other factors that are likely to contribute are the amount of time that it takes to maintain an active social media presence - time that could be spent either working towards the next paper or trying to keep a reasonable work-life balance - and the perceived lack of professional reward for this time, although this could be about to change with the introduction of unique identifiers for individual researchers (see http://about.orcid.org).
So what are the benefits against which these factors should be weighed when choosing whether to engage in social media activities? First, blogs, microblogs, podcasts and other forms of social media are rich sources of information, both in terms of the published literature and the latest news stories, and as they are user generated, the more people engage, the richer the information becomes. At a time when journal print editions have all but gone from the tables of departmental coffee rooms indeed, as the coffee rooms themselves are disappearing - social media have become an outlet for researchers to not only find out the latest information but also interact with their colleagues, both local and afar. Social media also provide the opportunity for researchers to extend their network of contacts.

In addition to such benefits, there are some potential risks associated with not engaging. For instance, blogs and microblogs are already a prominent place for discussing issues that affect all researchers, and advocates and campaigners are well adept at using a variety of social media resources to argue a particular point; if engagement with social media by the community remains low, then what may seem to be a commonly held view in the social media 'bubble' might in fact not be representative of the view held by the wider field but can still have a substantial impact on policy decisions.

There are numerous microbiologists among those who have trail-blazed the use of online tools to support their academic activities, and we fully support expanding this engagement to the rest of the microbiology community. We also appreciate that, for the uninitiated, knowing where to begin can be somewhat daunting, so we have posted links to a selection of microbiology-specific social media on our website to help you get started (http:// www.nature.com/nrmicro/info/info_social_media.html).

1. Cann, A., Dimitriou, K. \& Hooley, T. Social media: a guide for researchers Research Information Network [online] http://rinarchive jisc-collections.ac.uk/our-work/communicating-and-disseminatingresearch/social-media-guide-researchers (2011).

2. CIBER Research. Social media and research workflow. CIBER Research [online] http://www.ciber-research.eu/download/20101111-socialmedia-report.pdf (2011)

3. Carpenter, J. et al. Researchers of tomorrow: the research behaviour of generation Y doctoral students. JISC [online] http://www.jisc.ac.uk/ media/documents/publications/reports/2012/Researchers-ofTomorrow.pdf (2012)

4. Minocha, S. \& Petre, M. Handbook of social media for researchers and supervisors: digital technologies for research dialogues. Vitae [online] http://www.vitae.ac.uk/CMS/files/upload/Vitae_Innovate Open University_Social_Media_Handbook_2012.pdf (2012). 